\title{
Full Symmetry Groups and Exact Solutions to BKP and GKP Equations
}

\author{
Bo Ren ${ }^{1}$ and Jian-Yong Wang ${ }^{2}$ \\ ${ }^{1}$ Institute of Nonlinear Science, Shaoxing University, Shaoxing 312000, China \\ ${ }^{2}$ Department of Physics, Shanghai Jiao Tong University, Shanghai 200040, China \\ Correspondence should be addressed to Bo Ren; renbosemail@gmail.com
}

Received 1 July 2014; Accepted 9 September 2014; Published 18 September 2014

Academic Editor: Juan Jose Hernandez-Rey

Copyright (C) 2014 B. Ren and J.-Y. Wang. This is an open access article distributed under the Creative Commons Attribution License, which permits unrestricted use, distribution, and reproduction in any medium, provided the original work is properly cited.

We investigate the (2+1)-dimensional nonlinear BKP and GKP equations with the modified direct CK's method. Then, we get its Lie point groups and the full symmetry group, and a relationship is constructed between the new solutions and the old one. Based on the relationship, the new solutions can be obtained by using a given solution of the equations.

\section{Introduction}

Nonlinear partial differential equations are widely used to describe complex phenomena in various fields of science, especially physics. It is known that to find exact solutions of differential equations is always one of the central themes of perpetual interest in mathematics and physics. Many effective methods have been constructed to obtain the explicit exact solutions [1-5]. Among these methods, the Lie group techniques provide a very important method for obtaining exact solutions of nonlinear differential equations. Usually, the classical Lie group approach [6], the nonclassical Lie group approach [7], and the Clarkson-Kruskal (CK) direct method [8] are powerful methods. Recently, Lou and Ma proposed the modified direct method [9], which is inspired by CK's direct method and is an effective method to investigate the nonlinear equations. Symmetry groups and new exact solutions of many nonlinear systems are found by using the modified CK's direct method. The expression of the exact finite transformations of the Lie groups is much simpler than those obtained via the standard approaches. Therefore, the modified CK's direct method has been widely studied $[10,11]$, including the variable coefficient [12] and high-dimensional nonlinear systems $[13,14]$.

Simultaneously with the quest for the exact solutions, to find the new integrable nonlinear equations is very important work. Recently, using the sense of Kadomtsov and Petviashivilli, who relaxed the restriction that the waves be strictly one-dimensional in the Burgers and $\mathrm{KdV}$ equation, leads to the completely integrable Burgers Kadomtsev Petviashvili (BKP) and Gardner Kadomtsev Petviashvili (GKP) equations, respectively $[15,16]$. The exact soliton solutions of BKP and GKP equation have been studied with the Hirota's bilinear method $[17,18]$. Symmetries and the conservation laws to the GKP equation are also given by using the direct symmetry method [18]. In this paper, we will use the modified direct method to find non-Lie symmetry group and the new exact solutions of the BKP and GKP system.

The structure of this paper is organized as follows. The symmetry groups and Lie symmetry of the $(2+1)$-dimensional BKP and GKP equations are obtained using the modified CK's direct method, respectively, in Sections 2 and 3. Some new solutions are given with the symmetry group theorem and the known solutions. The last section is a simple summary and discussion.

\section{Symmetry Groups for (2+1)-Dimensional BKP Equation}

In this section we will study the BKP equation [15]:

$$
\left(u_{t}+u u_{x}+\mu u_{x x}\right)_{x}+\lambda u_{y y}=0 .
$$


According to the modified direct method [8], we set the solution of (1) in the form

$$
u(x, y, t)=\alpha+\beta U(X, Y, T),
$$

where $\alpha, \beta, X, Y$, and $T$ are functions of $(x, y, t)$ to be determined by requiring $U(X, Y, T)$ to satisfy the same $(2+1)$ dimensional BKP equation as $u(x, y, t)$ under the transformation $\{u, x, y, t\} \rightarrow\{U, X, Y, T\}$; that is,

$$
\left(U_{T}+U U_{X}+\mu U_{X X}\right)_{X}+\lambda U_{Y Y}=0 .
$$

Substituting (2) into (1) and requiring the coefficients of $U$ and its derivatives to be zero, we get

$$
\begin{gathered}
X=C \sqrt{C_{1}} x+\frac{G_{t}}{2 \lambda C_{1}^{1 / 4} \sqrt{C} A} y+F, \\
Y=\frac{C_{1}^{3 / 4} A}{\sqrt{C}} y+G, \quad T=C_{1}^{2} t+C_{2}, \\
\alpha=-\frac{\sqrt{C}}{2 \lambda C_{1}^{3 / 4} A} G_{t t} y-\frac{C}{4 \lambda C_{1}^{3 / 2}} G_{t}^{2}-\frac{C}{4 \lambda C_{1}^{3 / 2}}, \\
\beta=C \sqrt{C_{1}},
\end{gathered}
$$

where $C_{1}, C_{2}$ are arbitrary constants, $G, F$ are arbitrary functions of $t$, and $A^{2}=C^{2}=1$. Obviously, we have the following theorem.

Theorem 1. If $U(x, y, t)$ is a solution of $(2+1)$-dimensional $B K P(1)$, then

$$
u(x, y, t)=\alpha+\beta U(X, Y, T)
$$

is also a solution of (1), where $X, Y, T, \alpha$, and $\beta$ are satisfied (4). Due to the discrete values $A$ and $C$, the corresponding symmetry group is divided into four sectors

$$
\begin{array}{cc}
A=1, & C=1, \\
A=1, & C=-1, \\
A=-1, & C=1, \\
A=-1, & C=-1 .
\end{array}
$$

That is to say, the full symmetry group $\mathscr{G}_{B K P}$ for the BKP equation is the product of the connected Lie point symmetry group $\mathcal{S}$ and the discrete group $\mathscr{D}$; that is,

$$
\begin{gathered}
\mathscr{G}_{B K P}=\mathscr{D} \otimes \mathcal{S}, \\
\mathscr{D}=\left\{I, \sigma^{y} R, \sigma^{y}, R\right\},
\end{gathered}
$$

where I is the identity transformation and

$$
\begin{gathered}
\sigma^{y}: u(x, y, t) \longrightarrow u(x,-y, t), \\
R: u(x, y, t) \longrightarrow-u(-x, i y, t), \quad(\sqrt{-1}=i) .
\end{gathered}
$$

The Lie point symmetry group will be obtained by setting

$$
\begin{gathered}
C_{1}=1+\epsilon c_{1}, \quad C_{2}=\epsilon c_{2}, \\
G=t+\epsilon g(t), \quad F=\epsilon f(t), \quad C=A=1,
\end{gathered}
$$

with an infinitesimal parameter $\epsilon$; then (5) can be written as

$$
u=U+\epsilon \sigma(U) .
$$

We can obtain the symmetry of (1)

$$
\begin{aligned}
\sigma(U)= & \left(\frac{1}{2} c_{1} x+\frac{2 g(t)_{t}}{\lambda c_{1}} y+f(t)\right) U_{x} \\
& +\left(\frac{3}{4} c_{1} y+g(t)\right) U_{y}+\left(2 c_{1} t+c_{2}\right) U_{t}+\frac{1}{2} c_{1} U \\
& -\frac{3 g(t)_{t t}}{8 \lambda c_{1}} y-\frac{g(t)_{t}^{2}}{6 \lambda c_{1}}-\frac{1}{6 \lambda c_{1}} .
\end{aligned}
$$

The equivalent vector associated with $\sigma(U)$ of the above symmetry is given by

$$
\begin{aligned}
V= & \left(\frac{1}{2} c_{1} x+\frac{2 g(t)_{t}}{\lambda c_{1}} y+f(t)\right) \frac{\partial}{\partial x} \\
& +\left(\frac{3}{4} c_{1} y+g(t)\right) \frac{\partial}{\partial y}+\left(2 c_{1} t+c_{2}\right) \frac{\partial}{\partial t} \\
& +\left(-\frac{1}{2} c_{1} U+\frac{3 g(t)_{t t}}{8 \lambda c_{1}} y+\frac{g(t)_{t}^{2}}{6 \lambda c_{1}}+\frac{1}{6 \lambda c_{1}}\right) \frac{\partial}{\partial U} .
\end{aligned}
$$

Therefore, we can easily obtain the new solutions with a known exact solution. Many kinds of exact solutions of the $(2+1)$-dimensional BKP equation have been given by many authors. In the following, we present one example to illustrate it. Suppose the multiple-wave solution is given by [15]:

$$
u(x, y, t)=\frac{2 \nu \sum_{i=1}^{N} e^{k_{i} x+k_{i} y-\left(\nu k_{i}^{2}+\lambda k_{i}\right) t}}{1+\sum_{i=1}^{N} e^{k_{i} x+k_{i} y-\left(\nu k_{i}^{2}+\lambda k_{i}\right) t}}
$$

then, the solution of (1) can be read as

$$
u(x, y, t)=\alpha+\beta \frac{2 \nu \sum_{i=1}^{N} e^{k_{i} X+k_{i} Y-\left(v k_{i}^{2}+\lambda k_{i}\right) T}}{1+\sum_{i=1}^{N} e^{k_{i} X+k_{i} Y-\left(v k_{i}^{2}+\lambda k_{i}\right) T}},
$$

where $\alpha, \beta, X, Y$, and $T$ are determined by (4).

\section{Symmetry Groups for (2+1)-Dimensional GKP Equation}

Let us consider the following GKP equation in the form

$$
\left(v_{t}+6 v v_{x}+6 \delta v^{2} v_{x}+v_{x x x}\right)_{x}+v_{y y}=0,
$$

where $\delta$ is arbitrary constant. In the same way as in last section, the symmetry transformation ansatz has the form

$$
v(x, y, t)=\gamma+\rho V(\xi, \eta, \tau),
$$


where $\gamma, \rho, \xi, \eta$, and $\tau$ are functions of $(x, y, t)$ to be determined by requiring $V(\xi, \eta, \tau)$ to satisfy the same $(2+1)$ dimensional GKP equation as $v(x, y, t)$ under the transformation $\{v, x, y, t\} \rightarrow\{V, \xi, \eta, \tau\}$

$$
\left(V_{\tau}+6 V V_{\xi}+6 \delta V^{2} V_{\xi}+V_{\xi \xi \xi}\right)_{\xi}+V_{\eta \eta}=0
$$

With the coefficient of $V$ and its derivatives to be zero and solving these equations, we get

$$
\begin{gathered}
\xi=C C_{1}^{1 / 3} x+\frac{\sqrt{C} C_{3} A}{2 C_{1}^{1 / 3} y+C_{5},} \\
\eta=A C_{1}^{2 / 3} \sqrt{C} y+C_{3} t+C_{4}, \\
\tau=C_{1} t+C_{2}, \quad \gamma=-\frac{1}{2 \delta}+\frac{C_{1}^{1 / 3} C A}{2 \delta}, \quad \rho=A C C_{1}^{1 / 3},
\end{gathered}
$$

where $C_{i}(i=1, \ldots, 5)$ are arbitrary constants and the discrete values are determined by $A^{2}=1, C^{3}=1, C_{3}^{2}=$ $(18 / \delta)\left(C_{1}^{4 / 3} / C^{2}-C_{1}^{2}\right)$. Obviously, we have the following theorem.

Theorem 2. If $V(x, y, t)$ is a solution of (2+1)-dimensional GKP (15), then

$$
v(x, y, t)=\gamma+\rho V(\xi, \eta, \tau)
$$

is also a solution of (15), where $\xi, \eta, \tau, \alpha$, and $\beta$ are satisfied (18). Due to the discrete values $A$ and $C$, the corresponding symmetry group is divided into six sectors

$$
\begin{gathered}
A=1, \quad C=1, \\
A=1, \quad C=-\frac{1}{2}(1+i \sqrt{3}), \\
A=1, \quad C=-\frac{1}{2}(1-i \sqrt{3}), \\
A=-1, \quad C=1, \\
A=-1, \quad C=-\frac{1}{2}(1+i \sqrt{3}), \\
A=-1, \quad C=-\frac{1}{2}(1-i \sqrt{3}) .
\end{gathered}
$$

With product of the connected Lie point symmetry group $\mathcal{S}$ and the discrete group $\mathscr{D}$, the full symmetry group of the GKP equation is

$$
\begin{gathered}
\mathscr{G}_{G K P}=\mathscr{D} \otimes \mathcal{S}, \\
\mathscr{D}=\left\{I, Q_{1}, Q_{2}, \Sigma^{y}, \Sigma^{y} Q_{1}, \Sigma^{y} Q_{2}\right\},
\end{gathered}
$$

where

$$
\begin{aligned}
Q_{1}: & v(x, y, t) \\
\longrightarrow & -\frac{1}{2}(1+i \sqrt{3}) \\
& \times v\left(-\frac{1}{2}(1+i \sqrt{3}) x, \frac{1}{2}(1-i \sqrt{3}) y, t\right), \\
Q_{2}: & v(x, y, t) \\
& \longrightarrow-\frac{1}{2}(1+i \sqrt{3}) \\
& \times v\left(-\frac{1}{2}(1-i \sqrt{3}) x, \frac{1}{2}(1+i \sqrt{3}) y, t\right), \\
\Sigma^{y}: & v(x, y, t) \longrightarrow-v(x,-y, t) .
\end{aligned}
$$

If we set

$$
\begin{gathered}
C_{1}=1+\epsilon c_{1}, \quad C_{2}=\epsilon c_{2}, \quad C_{3}=1+\epsilon c_{3}, \\
C_{4}=\epsilon c_{4}, \quad C_{5}=\epsilon c_{5}, \quad C=A=1,
\end{gathered}
$$

with an infinitesimal parameter $\epsilon$, then (16) can be written as

$$
v=V+\epsilon \sigma(V)
$$

We can get the symmetry of (15)

$$
\begin{aligned}
\sigma(V)= & \left(\frac{1}{3} c_{1} x+\left(\frac{1}{6} c_{1}+\frac{1}{2} c_{3}\right) y+c_{5}\right) V_{x} \\
& +\left(\frac{2}{3} c_{1} y+c_{3} t+c_{4}\right) V_{y}+\left(c_{1} t+c_{2}\right) V_{t} \\
& +\frac{1}{3} c_{1} V-\frac{1}{2 \delta}\left(1-\frac{1}{3} c_{1}\right) .
\end{aligned}
$$

The equivalent vector associated with $\sigma(V)$ of the above symmetry is given by

$$
\begin{aligned}
V= & \left(\frac{1}{3} c_{1} x+\left(\frac{1}{6} c_{1}+\frac{1}{2} c_{3}\right) y+c_{5}\right) \frac{\partial}{\partial x} \\
& +\left(\frac{2}{3} c_{1} y+c_{3} t+c_{4}\right) \frac{\partial}{\partial y}+\left(c_{1} t+c_{2}\right) \frac{\partial}{\partial t} \\
& +\left(-\frac{1}{3} c_{1} V+\frac{1}{2 \delta}\left(1-\frac{1}{3} c_{1}\right)\right) \frac{\partial}{\partial V},
\end{aligned}
$$

which is the same as that obtained by the standard Lie approach [18].

We can also easily get the new solutions with a known exact solution using Theorem 2 . Here we just select one exact solution as an example. Suppose the traveling solution of the GKP equation is given by [18]

$$
v(x, y, t)=-\frac{1}{2 \delta} \pm \frac{\sqrt{-\delta l^{2}}}{\delta} \operatorname{sn}^{-1}(l x+p y, m),
$$


where $l$ and $p$ are arbitrary constants and $m$ is the modulus of the Jacobi elliptic function. With the help of Theorem 2, the new solution of (15) can be read as

$$
v(x, y, t)=\gamma+\rho\left(-\frac{1}{2 \delta} \pm \frac{\sqrt{-\delta l^{2}}}{\delta} \mathrm{sn}^{-1}(l \xi+p \eta, m)\right),
$$

where $\gamma, \rho, \xi$, and $\eta$ are determined by (18). Obviously, lots of new solutions to (15) can be obtained from the known solutions.

\section{Conclusion}

In this paper, applying the symmetry group direct method, the symmetry groups of the (2+1)-dimensional BKP and GKP equation are obtained, and the relationship between the new solution and the known one for the BKP and GKP equation is set up. Based on a given solution, one can construct other new solutions with the help of a group theorem, and one concrete solution is given as an example. At the same time, there are lots of new nonlinear equations being introduced into the nonlinear science and theoretical physics [19-21]. The modified CK's direct method is appropriate for solving these problems and future work on these aspects is worthy of studying.

\section{Conflict of Interests}

The authors declare that there is no conflict of interests regarding the publication of this paper.

\section{Acknowledgments}

This work was supported by the National Natural Science Foundation of China under Grant no. 11305106 and the Natural Science Foundation of Zhejiang Province of China Grant no. LQ13A050001.

\section{References}

[1] M. J. Ablowitz and P. A. Clarkson, Solitons, Nonlinear Evolution Equations and Inverse Scattering, vol. 149 of London Mathematical Society Lecture Note Series, Cambridge University Press, Cambridge, UK, 2000.

[2] R. Hirota, The Direct Method in Soliton Theory, Cambridge University Press, Cambridge, UK, 2004.

[3] C. S. Gardner, J. M. Greene, M. D. Kruskal, and R. M. Miura, "Method for solving the Korteweg-deVries equation," Physical Review Letters, vol. 19, no. 19, pp. 1095-1097, 1967.

[4] J. Lin, B. Ren, H. Li, and Y. Li, "Soliton solutions for two nonlinear partial differential equations using a Darboux transformation of the Lax pairs," Physical Review E: Statistical, Nonlinear, and Soft Matter Physics, vol. 77, no. 3, Article ID 036605, 2008.

[5] R. Bo and L. Ji, "Painlevé properties and exact solutions for the high-dimensional Schwartz Boussinesq equation," Chinese Physics B, vol. 18, no. 3, pp. 1161-1167, 2009.

[6] P. J. Olver, Application of Lie Group to Differential Equation, Springer, Berlin, Germany, 1986.
[7] G. W. Bluman and J. D. Cole, "The general similarity solution of the heat equation," Journal of Applied Mathematics and Mechanics, vol. 18, pp. 1025-1042, 1969.

[8] P. A. Clarkson and M. D. Kruskal, "New similarity reductions of the Boussinesq equation," Journal of Mathematical Physics, vol. 30, no. 10, pp. 2201-2213, 1989.

[9] S. Y. Lou and H. C. Ma, "Non-Lie symmetry groups of $(2+1)$ dimensional nonlinear systems obtained from a simple direct method," Journal of Physics A: Mathematical and General, vol. 38, no. 7, pp. L129-L137, 2005.

[10] B. Ren, X. J. Xu, and J. Lin, "Symmetry group and exact solutions for the $2+1$ dimensional Ablowitz-Kaup-NewellSegur equation," Journal of Mathematical Physics, vol. 50, Article ID 123505, 2009.

[11] B. Li, W. Ye, and Y. Chen, "Symmetry, full symmetry groups, and some exact solutions to a generalized Davey-Stewartson system," Journal of Mathematical Physics, vol. 49, no. 10, Article ID 103503, 2008.

[12] H. Ma, A. Deng, and Y. Wang, "Exact solution of a KdV equation with variable coefficients," Computers \& Mathematics with Applications, vol. 61, no. 8, pp. 2278-2280, 2011.

[13] L. Wang and Z. Dong, "Symmetry reduction and exact solutions of the $(3+1)$-dimensional breaking soliton equation," Communications in Theoretical Physics, vol. 50, no. 4, pp. 832-840, 2008.

[14] Z.-Z. Dong, C. Yong, and Y.-H. Lang, "Symmetry reduction and exact solutions of the (3+1)-dimensional Zakharov-Kuznetsov equation," Chinese Physics B, vol. 19, Article ID 090205, 2010.

[15] A. Wazwaz, "Multiple-front solutions for the BurgersKadomtsev-Petviashvili equation," Applied Mathematics and Computation, vol. 200, no. 1, pp. 437-443, 2008.

[16] A. M. Wazwaz, "Solitons and singular solitons for the GardnerKP equation," Applied Mathematics and Computation, vol. 204, no. 1, pp. 162-169, 2008.

[17] N. Taghizadeh, M. Mirzazadeh, and F. Farahrooz, "Exact soliton solutions of the modified KdV-KP equation and the BurgersKP equation by using the first integral method," Applied Mathematical Modelling, vol. 35, no. 8, pp. 3991-3997, 2011.

[18] B. Xu and X. Liu, "Classification, reduction, group invariant solutions and conservation laws of the Gardner-KP equation," Applied Mathematics and Computation, vol. 215, no. 3, pp. 12441250, 2009.

[19] B. Ren and J. Lin, "A new $(2+1)$-dimensional integrable equation," Communications in Theoretical Physics, vol. 51, no. 1, pp. 13-16, 2009.

[20] S. Y. Lou and X. B. Hu, "Infinitely many Lax pairs and symmetry constraints of the KP equation," Journal of Mathematical Physics, vol. 38, no. 12, pp. 6401-6427, 1997.

[21] A. Maccari, "The investigation into new integrable systems of equations in 2+1-dimensions," Journal of Mathematical Physics, vol. 44, no. 1, pp. 242-250, 2003. 

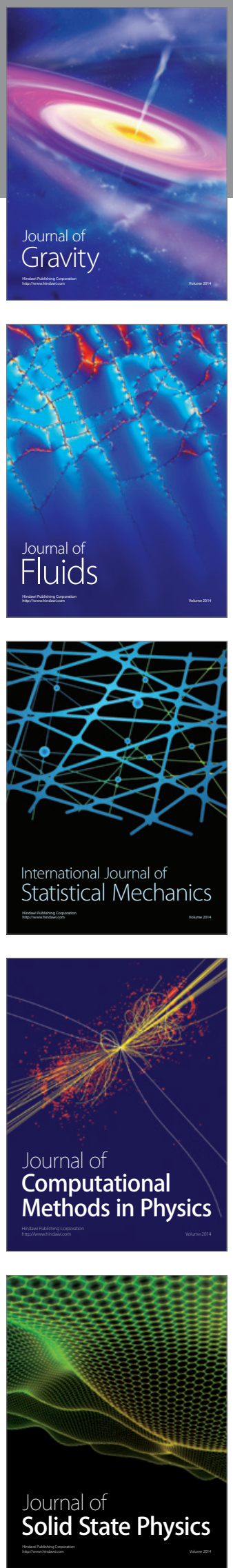

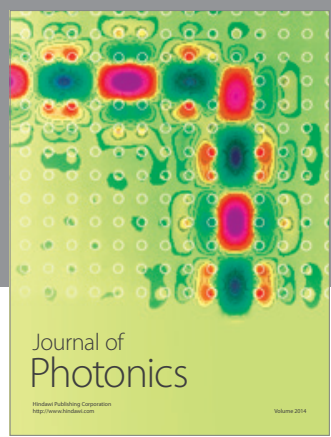

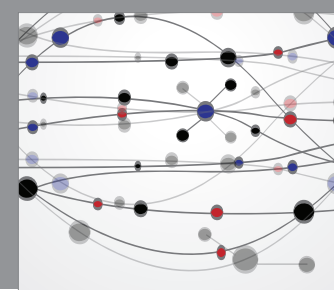

The Scientific World Journal

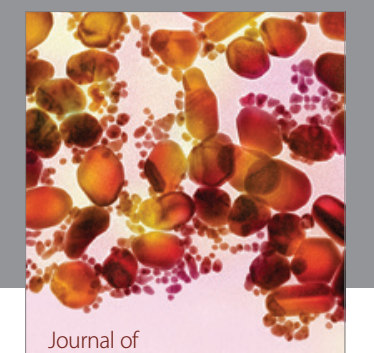

Soft Matter
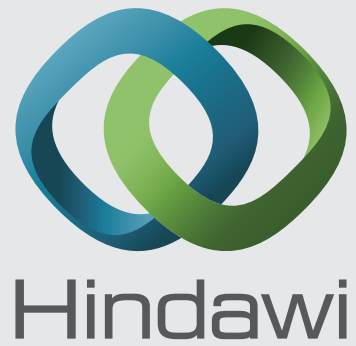

Submit your manuscripts at

http://www.hindawi.com
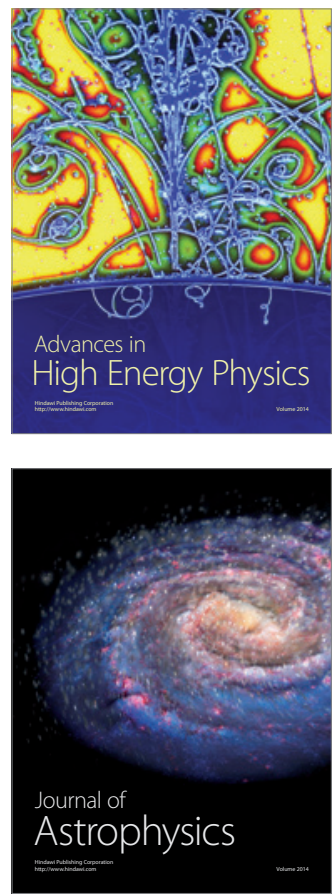
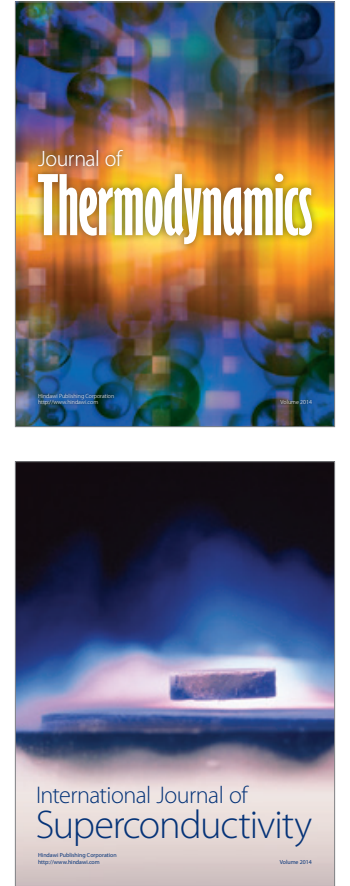
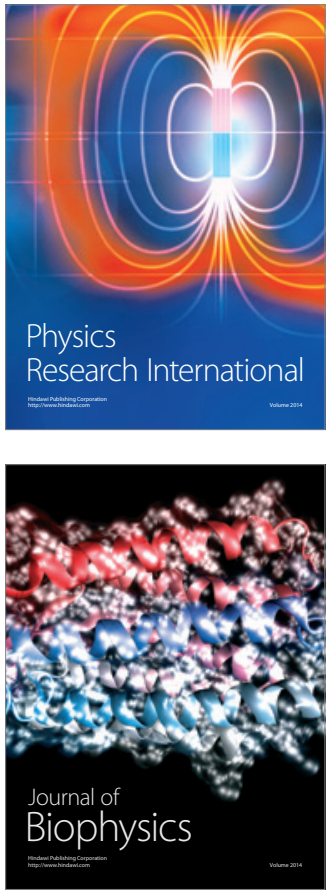
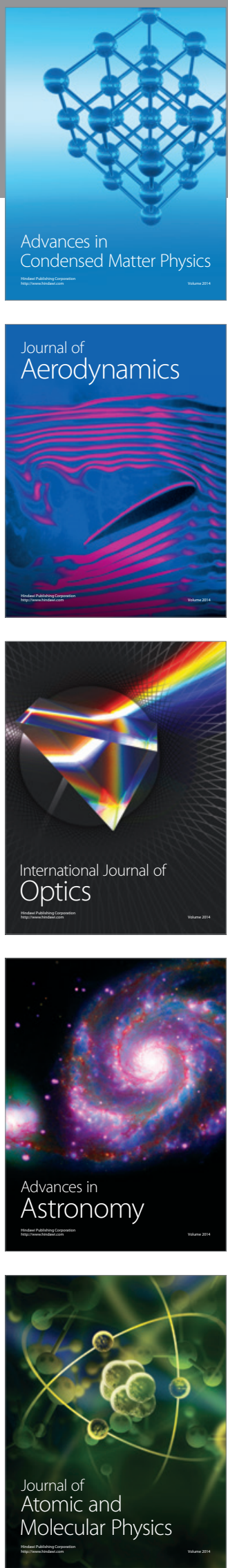